Please do not remove this page

RMIT

UNIVERSITY

\title{
Chromium single-photon emitters in diamond fabricated by ion implantation
}

Aharonovich, I; Castelletto, Stefania; Johnson, B; Simpson, David; Greentree, Andrew; Prawer, Steven https://researchrepository.rmit.edu.au/esploro/outputs/9921858163201341/filesAndLinks?institution=61 RMIT_INST\&index=null

Aharonovich, I., Castelletto, S., Johnson, B., Simpson, D., Greentree, A., \& Prawer, S. (2010). Chromium single-photon emitters in diamond fabricated by ion implantation. Physical Review B, 81(12), 1-4. https://doi.org/10.1103/PhysRevB.81.121201

Document Version: Published Version

Published Version: https://doi.org/10.1103/PhysRevB.81.121201

Repository homepage: https://researchrepository.rmit.edu.au

(c) 2010 The American Physical Society

Downloaded On 2023/04/26 10:33:56 +1000 


\section{DMIT \\ UNIVERSITY}

Thank you for downloading this document from the RMIT Research Repository.

The RMIT Research Repository is an open access database showcasing the research outputs of RMIT University researchers.

RMIT Research Repository: http://researchbank.rmit.edu.au/

\section{Citation:}

Aharonovich, I, Castelletto, S, Johnson, B, Simpson, D, Greentree, A and Prawer, S 2010, 'Chromium single-photon emitters in diamond fabricated by ion implantation', Physical Review B, vol. 81, no. 12, 121201, pp. 1-4.

See this record in the RMIT Research Repository at:

http://researchbank.rmit.edu.au/view/rmit:15494

Version: Published Version

Copyright Statement: (c) 2010 The American Physical Society

Link to Published Version:

http://dx.doi.org/10.1103/PhysRevB.81.121201

\section{PLEASE DO NOT REMOVE THIS PAGE}




\title{
Chromium single-photon emitters in diamond fabricated by ion implantation
}

\author{
Igor Aharonovich, ${ }^{*}$ Stefania Castelletto, Brett C. Johnson, Jeffrey C. McCallum, David A. Simpson, \\ Andrew D. Greentree, and Steven Prawer \\ School of Physics, University of Melbourne, Parkville, Victoria 3010, Australia \\ (Received 26 January 2010; revised manuscript received 18 February 2010; published 15 March 2010)
}

\begin{abstract}
Controlled fabrication and identification of bright single-photon emitters is at the heart of quantum optics. Here we demonstrate controlled engineering of a chromium bright single-photon source in bulk diamond by ion implantation. The $\mathrm{Cr}$ center has fully polarized emission with a zero-phonon line centered at $749 \mathrm{~nm}$, full width at half maximum of $4 \mathrm{~nm}$, an extremely short lifetime of $\sim 1 \mathrm{~ns}$, and a count rate of $0.5 \times 10^{6}$ counts $/ \mathrm{s}$. By combining the polarization measurements and the vibronic spectra, a model of the center has been proposed consisting of one interstitial chromium atom with a transition dipole along one of the $\langle 100\rangle$ directions.
\end{abstract}

DOI: 10.1103/PhysRevB.81.121201

Quantum technologies require new platforms and paradigms for their efficient fabrication and use. Single-photon sources exemplify this need, ${ }^{1}$ with their development pushing the bounds of existing materials and fabrication techniques. These sources are the main building blocks for quantum applications, such as quantum communications, ${ }^{2}$ quantum metrology, ${ }^{3}$ and quantum computation. Quantum dots $^{4}$ and single molecules ${ }^{5}$ have been utilized to generate single-photon emission; however, a promising class of room temperatures single-photon emitters (SPEs) are diamond color centers.

Color centers in monolithic single-crystal diamond are strong candidates for scalable quantum information processing (QIP) compared with those in nanocrystals, and hence several designs for large scale quantum architecture employing monolithic diamond have been proposed. ${ }^{6,7}$

The nitrogen-vacancy (NV) center is the most studied center for QIP applications owing to the availability of optical readout of the individual electronic spin state, ${ }^{8,9}$ leading to advanced applications such as nanomagnetometry. ${ }^{10,11}$ However, the NV center has fundamental limitations: strong phonon coupling of the excited state which results in a broad photoluminescence (PL) spectrum $(\sim 100 \mathrm{~nm})$ of which the zero-phonon line (ZPL) makes up $4 \%$. Emission of single photons in the ZPL is then extremely weak, typically on the order of a few thousands of photons per second. Such count rates are insufficient for the realization of advanced QIP protocols. These constraints motivate the need to identify and fabricate improved diamond based SPEs.

To move beyond the limitations of the NV center, alternative diamond based SPEs (Refs. 12 and 13) such as the silicon vacancy (Si-V) (Ref. 14), or the nickel related complex (NE8) (Ref. 15) have been investigated. However, the very low emission rates of the $\mathrm{Si}-\mathrm{V}$ center, and the difficulty to controllably fabricate both of these centers at the single center level, have motivated the search for alternative SPEs. A major challenge in the material science community in this search is to unambiguously identify new diamond based color centers and determine reproducible techniques to fabricate such sources in both bulk single-crystal diamond and nanodiamonds (NDs).

Circumstantial evidence of Cr-related centers in nanodiamond grown on sapphire substrates has been recently reported $;{ }^{16}$ however, unambiguous determination of its com-
PACS number(s): 81.05.ug, 42.50.Ar, 61.66.Dk

position and structure is yet to be ascertained. To explore the origins of novel color centers and their fundamental physical properties, the fabrication of these centers in monolithic single-crystal diamond rather than nanocrystals is necessary. Ion implantation ${ }^{17}$ offers the unique advantage generating optical centers based solely on the constituents used in the implantation process along with the known and wellcharacterized elements present in the bulk diamond. Implantation is therefore particularly advantageous when trying to identify the origin of an unknown color center.

Here we present and verify a class of single-photon emitters engineered in monolithic single-crystal diamond by the co-implantation of chromium and oxygen atoms. The ultrabright emission in the near infrared (NIR) at $749 \mathrm{~nm}$ and short excited state lifetime ( $\sim 1 \mathrm{~ns})$ surpass the performance of previously identified SPEs in single-crystal (bulk) diamond. Cr-related luminescence has been identified in sapphire ${ }^{18}$ and silicon. ${ }^{19}$ While the origin of the luminescence in silicon is still under investigation, ${ }^{20}$ the atomic structure of the center in sapphire is attributed to a substitutional $\mathrm{Cr}^{3+}$ ion. With this in mind, the co-implantation of $\mathrm{Cr}$ and $\mathrm{O}$ atoms into single-crystal diamond was performed to modify the oxidation state of the chromium in the diamond lattice.

Chemical vapor deposition (cvd) single-crystal diamond samples (type IIA, $[N]<1 \mathrm{ppm},[B]<0.05 \mathrm{ppm}$, element six) were co-implanted with $50 \mathrm{keV}$ chromium and $19.5 \mathrm{keV}$ oxygen to a fluencies of $1 \times 10^{11} \mathrm{Cr} / \mathrm{cm}^{2}$ and 1.5 $\times 10^{11} \mathrm{O} / \mathrm{cm}^{2}$, respectively. Note that these doses are below the limit of diamond graphitization. The implantation energies were chosen to maximize the proximity of the two atomic species. Figure 1(a) shows a Monte Carlo simulation using the stopping range of ions in matter (SRIM) software of implanted $\mathrm{Cr}$ and $\mathrm{O}$ atoms accelerated to 50 and $19.5 \mathrm{keV}$, respectively. The projected range of these implantations was approximately $25 \mathrm{~nm}$ below the diamond surface which allowed efficient out-coupling of the light emitted from the center. After the implantation, the diamonds were annealed at $1000{ }^{\circ} \mathrm{C}$ in a forming gas ambient $\left(95 \%-\mathrm{Ar}-5 \% \mathrm{H}_{2}\right)$ for $1 \mathrm{~h}$. Note that the annealing step applied after the implantation $\left(1000{ }^{\circ} \mathrm{C}\right)$ is not sufficient to cause any diffusion of $\mathrm{Cr}$ atoms in the stiff diamond lattice; $;^{21,22}$ hence, the formed centers are indeed located in the depth of the implantation.

Figure 1(b) shows a fluorescence confocal map recorded 

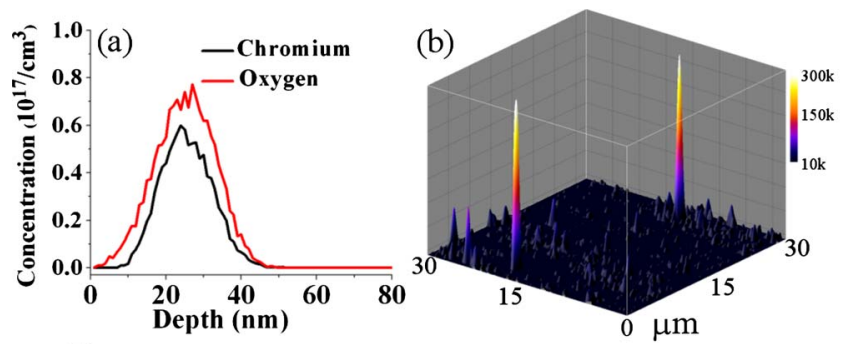

(c)

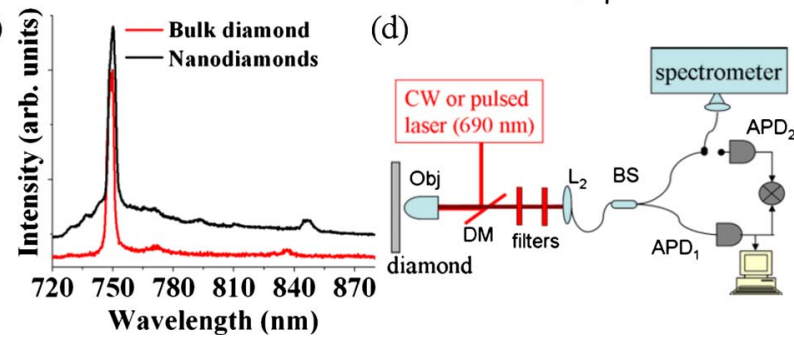

FIG. 1. (Color online) Chromium implanted diamond. (a) Monte Carlo simulation of $\mathrm{Cr}$ and $\mathrm{O}$ distribution inside a diamond following $50 \mathrm{keV} \mathrm{Cr}$ and $19.5 \mathrm{keV} \mathrm{O}$ implantation. (b) A confocal map recorded from the diamond sample co-implanted with $1 \times 10^{11} \mathrm{Cr} / \mathrm{cm}^{2}$ and $1.5 \times 10^{11} \mathrm{O} / \mathrm{cm}^{2}$. The bright spots correspond to $\mathrm{Cr}$ centers in diamond (c) PL spectrum recorded at room temperature from a bright spot on the confocal map shown in Fig. 1(b) red curve (dark gray). The black curve is the PL spectrum recorded from nanodiamonds grown on sapphire following the procedure reported elsewhere. ${ }^{15}$ The data from the nanodiamonds was displaced vertically for clarity. (d) The optical setup employed to characterize the samples.

from the (100) oriented single-crystal diamond after the coimplantation of chromium and oxygen. The bright spots in the confocal image correspond to the engineered centers from the ion implantation process.

Figure 1(c) shows a narrow PL spectrum, recorded at room temperature using a continuous-wave $(\mathrm{CW})$ diode laser emitting at $682 \mathrm{~nm}$ from one of the bright spots on the confocal map shown in Fig. 1(a) [red curve (dark gray)], with a ZPL centered at $749 \mathrm{~nm}$ and a full width at half maximum (FWHM) of $4 \mathrm{~nm}$. Similar PL lines were consistently observed from different bright spots across the sample. The co-implantation technique yielded 2-4 $\mathrm{Cr}$ centers with a ZPL centered at $749 \mathrm{~nm}$ per $50 \mu \mathrm{m}^{2}$, resulting in a conversion efficiency of less than $0.1 \%$. The black curve in Fig. 1(c) shows as a comparison a PL spectrum recorded from a center in nanodiamond grown on a sapphire substrate. ${ }^{16}$ The excellent agreement between the PL spectra, in terms of the ZPL and FWHM, in bulk diamond and NDs clearly indicates that these class of emitters can be attributed to $\mathrm{Cr}$.

The photon statistics of the PL in single-crystal diamond were studied by recording the second-order correlation function, $g^{(2)}(\tau)=\langle I(t) I(t+\tau)\rangle /\langle I(t)\rangle^{2}$, using a fiber-based Brown and Twiss setup. ${ }^{23}$ Figure 2 (a) shows the corresponding, $g^{(2)}(\tau)$ function, for the $749 \mathrm{~nm}$ center. The dip at zero delay time $g^{(2)}(\tau=0)=0.2$ is characteristic of non classical light and verifies single-photon emission. The deviation from zero at $\tau=0$ is attributed to the background within the singlecrystal diamond. The photon bunching $\left(g^{(2)}(\tau)>1\right)$ observed at longer delay times is indicative of a three level system
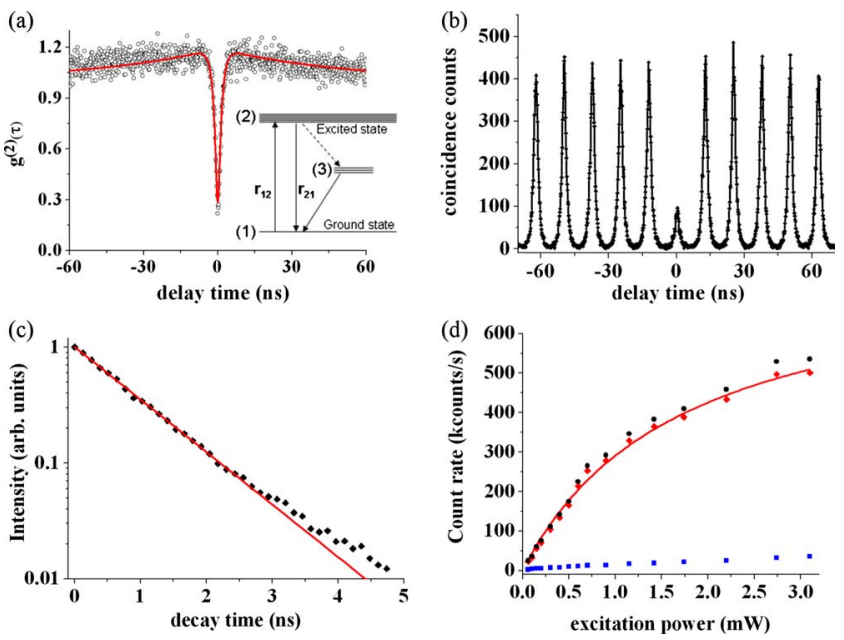

FIG. 2. (Color online) Optical characterization of the Cr center. (a) A normalized second-order correlation function, $g^{(2)}(\tau)$, corresponding to the PL line shown in Fig. 1(c), demonstrating singlephoton emission $\left(g^{(2)}(0)=0.2\right)$. The bunching $\left[g^{(2)}(\tau)>1\right]$ indicates of a presence of a third metastable state. The red (dark gray) line is the theoretical fit based on a three level model, taking into account the time response of the detectors. Inset, is a schematic model of a three level system. (b) Triggered SP emission is verified by exciting with a pulsed laser at $80 \mathrm{MHz}$. The deviation from zero of the $g^{(2)}(0)$ under CW excitation is attributed to the background and the time response jitter of the photo detectors and correlation electronics, while in the pulsed regime the deviation from zero can be attributed to background due to non perfect polarization condition of the pulsed excitation laser. (c) A direct lifetime measurement (dots) with a fit of a single exponential [red (dark gray) curve]. A lifetime of $1 \pm 0.1 \mathrm{~ns}$ is deduced from the fit. (d) Single-photon emission count rate recorded from the same emitter as a function of excitation power. The squares represent the background noise; the black circles represent the raw data and the red (dark gray) rhombs represent the background corrected count rate. The fit (red curve) was obtained from the solution of a three level system.

with a long-lived metastable state. ${ }^{8,15}$ To verify single-photon emission on demand, a key feature for many quantum optical applications, the center was excited with a $690 \mathrm{~nm}$ pulsed laser with a repetition rate of $80 \mathrm{MHz}$, as shown in Fig. 2(b). The vanishing peak at $\tau=0$ indicates that only one photon is emitted per excitation pulse. The excited state lifetime of the same emitter is shown in Fig. 2(c) and was obtained under pulsed excitation at $40 \mathrm{MHz}$. From the single-exponential fit to the fluorescence decay, an excited state lifetime of $1.0 \pm 0.1 \mathrm{~ns}$ is deduced. This is a short fluorescence lifetime when compared to other known SP emitters in diamond, 8,15 indicative of a very strong dipole moment in the radiative transition, which we have determined to be $60 \mathrm{D}$. The measured lifetime is in agreement with the one measured for the center in nanodiamonds $(1.1 \pm 0.1 \mathrm{~ns})$.

To quantify the single-photon efficiency, the count rate was measured as a function of the excitation power. Figure 2(d) shows the measured single-photon count rate as a function of excitation power. A measured count rate of $\sim 0.5$ $\times 10^{6}$ counts/s makes the $\mathrm{Cr}$ center the brightest reported single-photon source in a monolithic single-crystal diamond to date and within an order of magnitude of the brightest 

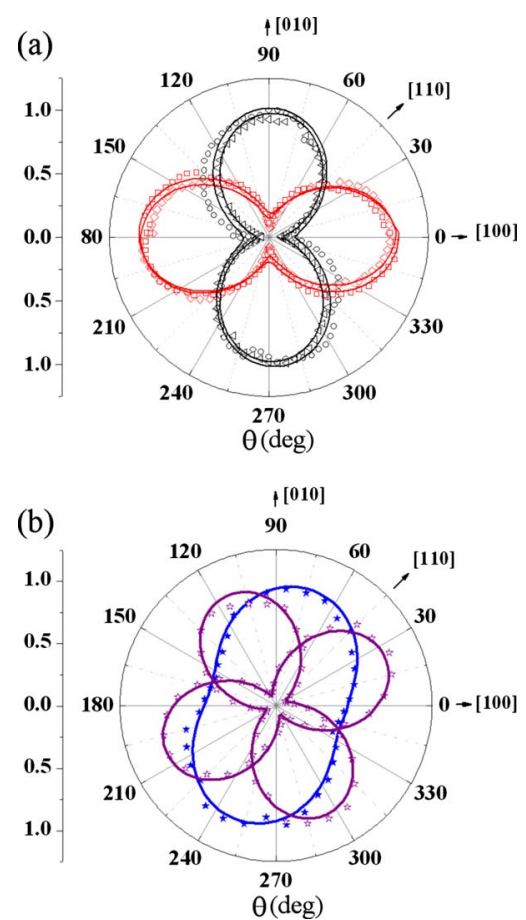

FIG. 3. (Color online) Polarization properties and the structure of the Cr center. (a) Polarization measurements of the $749 \mathrm{~nm}$ emitter represented in polar coordinates. $\theta$ is the polarization angle referred to the main crystallographic axes. PL intensity versus polarization of the excitation laser for four different SP emitters (black triangles, black circles, red (dark gray) squares and red (dark gray) diamonds) with a ZPL centered at $749 \mathrm{~nm}$. An extinction of the signal down to the typical background level is demonstrated. A period of nearly $90^{\circ}$ between the maximum and the minimum of different emitters [black and red (dark gray] colors) is observed. (b) Modulation of the emission intensity measured by rotating a linear polarization analyzer at the detection channel, with a fixed polarization of the excitation field [blue (black) filled stars]. By introducing a quarter-wave plate before the analyzer, full polarization contrast was achieved. Purple (gray) empty stars show two possible polarization emissions for two different wave plate positions.

nanodiamond sources. ${ }^{12,16}$ Furthermore, when these centers are integrated with the recent diamond based antennas, ${ }^{24}$ even higher count rates can be expected. Note that the center is located only a few tens of nm below the diamond surface which is advantageous for efficient coupling of the emitted light to external cavities or waveguides. ${ }^{25}$

To understand the dipole orientation of the $\mathrm{Cr}$ center relative to the crystal axis, excitation polarization measurements on the emitters with a ZPL centered at $749 \mathrm{~nm}$ were performed. ${ }^{26,27}$ Prior to each measurement, the antibunching was recorded to verify that only single emitters were addressed. All single $749 \mathrm{~nm}$ centers studied here were found to exhibit a dependence on the incident excitation polarization. Two distinct polarization dependencies were identified by characterizing numerous single $749 \mathrm{~nm}$ emitters and these are shown in Fig. 3(a) for four separate single emitters. By varying the incident polarization angle by $90^{\circ}$ in the respective configuration the PL intensity evolves from maximum to minimum intensity, with extinction ratios of $96 \%$, as seen in
Fig. 3(a). This is consistent with the characteristic dipole transition behavior.

Given that the diamond sample is (100) oriented, the direction of the center transition dipole moment may be determined. The electric field of the incoming laser is parallel to the (100) plane of the crystal. Since the modulation of the excitation polarization angle results in an absolute extinction of the PL, the dipole of the center must be aligned in the $[0 y z]$ direction, otherwise full extinction of the PL would not be observed. Furthermore, the period of the minima (or the maxima) of the emitters is $90^{\circ}$, which indicates that only two different projections of the dipole on the (100) plane are possible. Due to the diamond structure, the only crystallographic directions that meet these conditions are the [010] or [001]. Note that the dipole of the center can be also aligned along the [100] direction; however, in this case it will always be perpendicular to the excitation electric field and thus, will never be excited. Thus, the transition dipole moment of the observed centers is aligned in one of the $\langle 100\rangle$ directions.

The emission polarization of the system was studied by fixing the polarization excitation to maximum and rotating the polarizer at the detection channel. The nonperfect degree of polarization of the emitted light [Fig. 3(b), blue full stars] may be attributed to the fact that the excitation and the absorption dipoles are not parallel. The difference between the absorption and excitation dipole orientation is due to a redistribution of the electrons upon photon absorption.

Upon introduction of a quarter-wave plate in the detection channel before the analyzer, a full extinction of the emitted light [Fig. 3(b) purple open stars] was observed, confirming that the emitted light is polarized. Different orientations of the modulated emission were obtained by varying the quarter-wave plate position, as shown in Fig. 3(b) for two different positions (recorded from the same emitter). The angle between the maxima of the emitted light and the cube main axis does not give information of the relative orientation of the emission and absorption dipoles, which requires more accurate evaluation of the anisotropy using polarization microscopy techniques. ${ }^{28}$ The fully polarized light indicates however that transitions such as thermal relaxation within the excited state manifold do not exist under our experimental conditions and the decay to the ground state occurs from an individual excited state level. This is an important characteristic for the generation of indistinguishable single photons and quantum entanglement. ${ }^{29}$

Finally, we discuss the atomic structure of the $\mathrm{Cr}$ center in diamond. This center was fabricated only when $\mathrm{Cr}$ implantation into diamond was employed. A substitutional atom in a diamond lattice exhibits tetrahedral symmetry and would possess a different excitation polarization dependence than the observed here. Furthermore, most of the substitutional atoms in the diamond structure form complexes aligned along the $\langle 111\rangle$ axis (e.g., substitutional nitrogen adjacent to a vacancy). As the excitation polarization measurements in this work result in a complete extinction of the PL and indicate that the excitation dipole of the center is located on the $\{001\}$ planes, the incorporation of $\mathrm{Cr}$ atoms as substitutional is excluded. Similarly, an adjacent vacancy, which generally occupies the (111) location site, is also excluded. Since the size of the $\mathrm{Cr}$ atom is significantly larger than the carbon 
matrix, an interstitial location is most stable. We therefore conclude that the $\mathrm{Cr}$ atom occupies an interstitial related site, with a possible distortion of a surrounding diamond lattice.

We then investigated the effects of local vibrations due to the number of atoms forming the defect. A defect consisting of an impurity atom of mass $M_{I}$ located in a matrix with host atom mass $M_{C}$, generates quasilocal vibrations which can be described by the following equation: $\omega_{Q L}=\omega_{D} \sqrt{M_{C} / 3\left(k M_{I}-M_{C}\right)}$ where $\omega_{D}=150 \mathrm{meV}$ is the Debye frequency of the diamond lattice and $k$ is the number of impurity atoms involved in the quasilocal vibration. ${ }^{30}$ For one $\mathrm{Cr}$ atom, the vibration should occur $47 \mathrm{meV}$ from the ZPL, which in the present case occurs at $771 \mathrm{~nm}$. Following the measured PL from the bulk diamond presented in Fig. 1(c), there is good agreement with the theory for one $\mathrm{Cr}$ atom as a vibronic feature can be observed at $771 \mathrm{~nm}$, which corresponds to $47 \mathrm{meV}$ shift from the ZPL. We propose the name UM2 for this center

We cannot at this stage unambiguously identify the role of the oxygen in the co-implantation except to note that the co-implantation significantly enhances the formation of the UM2 center; one may assume that its behavior as an electron trap may set the charge state of the chromium. A test implantation of chromium only into the same type of single-crystal diamond, followed by the same annealing treatment, yielded only limited PL lines centered at $749 \mathrm{~nm}$. The ratio of UM2 centers observed in the co-implantation of chromium and oxygen compared to the chromium only implantation was $\sim 10: 1$.

In summary, we demonstrate the controlled engineering of a chromium bright single-photon source in bulk diamond, labeled as UM2. The center located in close proximity to the diamond surface $(\sim 25 \mathrm{~nm})$, has fully polarized emission with a ZPL centered at $749 \mathrm{~nm}$, FWHM of $4 \mathrm{~nm}$, an extremely short lifetime of $1 \mathrm{~ns}$, and exhibits a count rate of approximately $0.5 \times 10^{6}$ counts/s, making it the brightest SPE in bulk diamond to date. The UM2 center exhibits an absorption dipole along one of the $\langle 100\rangle$ directions and most likely consisting of one interstitial chromium atom. The enhanced properties of this center compared with the known diamond centers and the scalable fabrication technique suggest that the UM2 center will be important for a range of applications including quantum metrology, quantum cryptography, and biomarking.

The authors thank Faruque Hossain and Fedor Jelezko for many helpful discussions. This work was supported by the Australian Research Council, The International Science Linkages Program of the Australian Department of Innovation, Industry, Science and Research (Project No. CG110039) and by the European Union Sixth Framework Program under Program No. EQUIND IST-034368. A.D.G. was supported by the Australian Research Council Queen Elizabeth II Foundation (Project No. DP0880466).
*Corresponding author; i.aharonovich@pgrad.unimelb.edu.au

${ }^{1}$ A. J. Shields, Nat. Photonics 1, 215 (2007).

${ }^{2}$ N. Gisin, G. G. Ribordy, W. Tittel, and H. Zbinden, Rev. Mod. Phys. 74, 145 (2002).

${ }^{3}$ V. Giovannetti, S. Lloyd, and L. Maccone, Phys. Rev. Lett. 96, 010401 (2006).

${ }^{4}$ C. Santori, M. Pelton, G. Solomon, Y. Dale, and Y. Yamamoto, Phys. Rev. Lett. 86, 1502 (2001)

${ }^{5}$ B. Lounis and W. E. Moerner, Nature (London) 407, 491 (2000).

${ }^{6}$ M. V. G. Dutt, L. Childress, L. Jiang, E. Togan, J. Maze, F. Jelezko, A. S. Zibrov, P. R. Hemmer, and M. D. Lukin, Science 316, 1312 (2007).

${ }^{7}$ A. M. Stephens, Z. W. E. Evans, S. J. Devitt, A. D. Greentree, A. G. Fowler, W. J. Munro, J. L. O'Brien, K. Nemoto, and L. C. L. Hollenberg, Phys. Rev. A 78, 032318 (2008).

${ }^{8}$ F. Jelezko and J. Wrachtrup, Phys. Status Solidi A 203, 3207 (2006).

${ }^{9}$ R. Hanson, V. V. Dobrovitski, A. E. Feiguin, O. Gywat, and D. D. Awschalom, Science 320, 352 (2008).

${ }^{10}$ J. R. Maze et al., Nature (London) 455, 644 (2008).

${ }^{11}$ G. Balasubramanian et al., Nature (London) 455, 648 (2008).

${ }^{12}$ D. A. Simpson, E. Ampem-Lassen, B. C. Gibson, S. Trpkovski, F. M. Hossain, S. T. Huntington, A. D. Greentree, L. C. L. Hollenberg, and S. Prawer, Appl. Phys. Lett. 94, 203107 (2009).

${ }^{13}$ D. B. Naydenov, R. Kolesov, A. Batalov, J. Meijer, S. Pezzagna, D. Rogalla, F. Jelezko, and J. Wrachtrup, Appl. Phys. Lett. 95, 181109 (2009).

${ }^{14}$ C. L. Wang, C. Kurtsiefer, H. Weinfurter, and B. Burchard, J. Phys. B 39, 37 (2006).

${ }^{15}$ T. Gaebel, I. Popa, A. Gruber, M. Domhan, F. Jelezko, and
J. Wrachtrup, New J. Phys. 6, 98 (2004).

${ }^{16}$ I. Aharonovich, S. Castelletto, D. A. Simpson, A. Stacey, J. McCallum, A. D. Greentree, and S. Prawer, Nano Lett. 9, 3191 (2009).

${ }^{17}$ J. R. Rabeau et al., Appl. Phys. Lett. 88, 023113 (2006).

${ }^{18}$ T. M. Hensen, M. J. A. de Dood, and A. Polman, J. Appl. Phys. 88, 5142 (2000).

${ }^{19}$ H. Conzelmann, K. Graff, and E. R. Weber, Appl. Phys. A: Mater. Sci. Process. 30, 169 (1983).

${ }^{20}$ J. Schmidt, R. Krain, K. Bothe, G. Pensl, and S. Beljakowa, J. Appl. Phys. 102, 123701 (2007).

${ }^{21}$ A. Zaitsev, Optical Properties of Diamond. A Data Handbook (Springer, Berlin, 2001).

${ }^{22}$ K. Iakoubovskii and A. T. Collins, J. Phys.: Condens. Matter 16, 6897 (2004).

${ }^{23}$ R. H. Brown and R. Q. Twiss, Nature (London) 177, 27 (1956).

${ }^{24}$ T. M. Babinec, B. J. M. Hausmann, M. Khan, Y. Zhang, J. R. Maze, P. R. Hemmer, and M. Loncar, Nat. Nanotechnol. 5, 195 (2010).

${ }^{25}$ K. M. C. Fu, C. Santori, P. E. Barclay, I. Aharonovich, S. Prawer, N. Meyer, A. M. Holm, and R. G. Beausoleil, Appl. Phys. Lett. 93, 234107 (2008).

${ }^{26}$ R. J. Epstein, F. M. Mendoza, Y. K. Kato, and D. D. Awschalom, Nat. Phys. 1, 94 (2005).

${ }^{27}$ T. P. M. Alegre, C. Santori, G. Medeiros-Ribeiro, and R. G. Beausoleil, Phys. Rev. B 76, 165205 (2007).

${ }^{28}$ S. A. Empedocles, R. Neuhauser, and M. G. Bawendi, Nature (London) 399, 126 (1999).

${ }^{29}$ P. Kok, W. J. Munro, K. Nemoto, T. C. Ralph, J. P. Dowling, and G. J. Milburn, Rev. Mod. Phys. 79, 135 (2007).

${ }^{30}$ A. M. Zaitsev, Phys. Rev. B 61, 12909 (2000). 